\title{
The Concept of Yin and Yang and Ancient Literature
}

\author{
Xie Guorong \\ Guilin Institute of Tourism \\ Guilin, Guangxi, 541006 China
}

\begin{abstract}
The relationship between the concept of Yin and Yang and ancient literature is rarely discussed by people currently. This paper makes an initial analysis of concept of Yin and Yang and ancient literature relationship from three aspects of combination, change and neutralization. In literature creation, the universal view of Yin and Yang lift, things having two and only two causes that ancient scholars focus on Pianli creation tendency, which is the deep cultural reason of ancient prose development and ancient literature language form generally antitheses. In literature change aspect, the natural change view of "things having two" and "only two" causes that ancient people take natural change to explain literature development change phenomenon, forming Liu Xie as the representative "articles changing and without losing normal state" literature development view. In literature art aesthetics aspect, Yin and Yang, Cliffside and auspicious fundamental theory causes ancient people to take "neutral" as the beauty phenomenon, making "neutral" beauty theory become the ancient aesthetics trend for thousands of years.
\end{abstract}

Keywords: ancient literature; ancient philosophy; the concept of Yin and Yang;

\section{INTRODUCTION}

For a long time, people focus on the relationship between the concept of Yin and Yang, ancient philosophy, politics and medicine, but few people analyze the relationship between concept of Yin and Yang and literature, which makes people sad. This paper starts from three aspects of antitheses, change and neutralization, and it makes further analysis of concept of Yin and Yang and ancient literature, in order to propose the problems.

\section{FOCUSING ON PIANLI CREATION TENDENCY}

Concept of Yin and Yang divides things in the world abstract as Yin and Yang of two categories, thinking that all the things in the world, death or life, are touched and swings and sympathetic by Yin and Yang of two forces.

Yin and Yang lift, "things having two, and only two" universal view and its thinking mode influence Chinese culture deeply, and its influence on Chinese ancient literature form has the "natural pair" couplets, leading to the common parallel of Chinese ancient literature language form, namely, forming Pianli creation tendency.

On the prose, scholars advocate "Yiji", "Bianyan" and "Chinese single tone". Why do the same language system, the same single tone and meaning character, only China has parallel prose poetic couplet, others are not? What is the inevitable factor? This is "things having two, and only two" Yin and Yang lift concept.

Liu Xie was first to see the problem. Liu said in "Wenxin craving the dragon", "creator shaped, body will double, god is not isolated." So, "heart diction" is "natural pair".

"Things having two, and only two" Yin and Yang lift thinking not only leads to ancients in advocating antithesis of customs, namely, it leads to the appearance of Pian poems (including poetic couplet) generation and development, and it also leads to general antithesis of Chinese ancient literature language form.

\section{PAYING ATtention to ChANGE DEVElOPMENT}

\section{CONCEPT}

Yin and Yang philosophy is a variation science. Early in the book "change" old testament, it has "no flat no slope, no return no back". (Tai hexagram, speech", "repeated speech, seven days back" (repeated speech) speech, and then "Yizhuan" clearly proposes "Yi" is book unsettled, as are repeatedly moved, about six empty, the upper and lower volatile, adaptability. "copulative" below emphasizes "life is easy" (copulative". This shows that since the creation of Yin and Yang philosophy, it focuses on change and development. But due to Yin and Yang concept originates from ancient worship, analogy and simulation of the universe, or humanity itself. Thus, its change will have natural cycle theory color, becoming a clockwise impartial, keeping the right, not wrong, and this cycle of metaphysical distinctive features.

The holder of this literature development view is mainly Liu Xie. Why do Liu Xie propose this viewpoint? We need to contact his Tao theory to see it clearly. "Wenxin craving dragon" first lists "Yuandao", "Zheng Sheng" and "classics" of three chapters, making sky, nature and human combine in one, taking the whole book as the general principle. The so-called "Tao" mainly refers to the heaven and nature. He thinks in "original" article, "the sun and the moon", "mountain and water" is "Tao". Thus, he obtains the conclusion of "the heaven and the earth" is the so-called "human". In Liu Xie's view, human is "soul of the clock", "five lines for the show, the real heart of the heaven and earth", and we can exist by side with heaven and earth, coherent and divided into three parts of the universe as a whole. Since man is the heart of the heaven and earth, you 
should know the nature of the heaven and earth, and this is only the finest people---saint to do. In Liu Xie's view, the sage can refer to the sun and the moon, good God and rules, thinking conforming to agreement". (Zhengsheng). In other words, the sage knows heaven and earth, and saint conforms to heaven. And "Tao" is only one, and it is eternal. Thus, to clarify the "Tao" scriptures (sage paper) is the enduring to the road, not the issue of Hongjiao". (Zhengsheng).

Thus, Ming dynasty $\mathrm{Hu}$ Yinglin "air migration" and Qing dynasty Ye Xie's "literature and Tao change", develop based on Liu Xie's literature development view thought. Hu's "Shisou" takes out "air migration" to explain the change development of literature, thinking "articles about air migration", not human act. "Gas" refers to the life of the times, but also it is with a thin layer of heaven color. And heaven is Yin and Yang moving and four seasons variable. Qing dynasty Ye xie clearly proposes literature change is from heaven "Tao" change in "original poem".

\section{MARKING "NeUtralization" AESTHETIC TREND}

Ancient "neutralization" beauty theory development process mainly experiences three stages.

First, Yin and Yang concept "obtaining is lucky" idea leads to middle course of the Confucian. Confucius was the first to propose the "doctrine of the mean" theory, and also the first to use it to evaluate literature and art people. "Quality wins literature is wild, and literature wins quality is history. Gentle is gentlemen." (Analects of Confucius). "Guanju" "joyous but not indecent, mournful but not distressing.(Analects of Cnfucius, eight Yi), it is the typical neutralization beauty theory. In the book of rites, neutral is to the fundamental cognition to understand the world. In the book of rites, music, the writer often takes ritual and music, thinking that music role is the same, and ritual is the role of difference. He thinks that "music is made by heaven, and ritual is by earth, and otherwise it is chaotic, as it bursts. And it must be innocent, conforming to neutralization standard. However, "the book of rites" further uses Yin and Yang to illustrate the idea of "courtesy distinction and music the same". It takes heaven above and earth below, high and low, is it ritual. The friction of Yin and Yang, swinging and all things emerging is music. So for further reference: music means Yang, and ritual means Yin. "Ritual is upper and music is lower" (ritual). "Yin and Yang are all things." (sacrifice). He thinks that the ritual and music making is from Yin and Yang, and Yin and Yang are neutral to produce all things. So, neutralization is the basis of heaven and earth. The so-called "passion not often, but all of that. Neutralization is the foundation of the heaven, and the goal of the heaven. Courtesy, music, poetry, books and even all the culture should reflect the neutral idea. Because "neutralization concept has the basic argument of heaven, making the "neutralization" beauty thought is accepted by most people, in order to form a huge flow. But, "neutralization" theory before Qin and Han dynasty focuses on thought content, mainly in emotion and reason neutralization, with strong political and humanity color.
Second, Liu Xie in Wei and Jin era greatly widened neutralization beauty theory range. After that, metaphysics prosperity declines of Confucian classics, instead, more from aesthetics angle, it discusses "neutralization" idea literature art criticism theory. Liu Xie is the representative of this aspect. Liu Xie proposes "character" theory in "Wenxin craving dragon", focusing on "masculine" beauty. But from the overall view, he is not just for rigid but for applicable. (set), focusing on rigid and soft and neutral, and in many issues, it insists on neutral view.

Third, Tang Dynasty monk Jiaoran basically picks out "neutral" beauty theory politics ethical color, and from pure beauty angle, it illustrates "neutralization" beauty. Jiao ran points out in "poetry", "four no", "two yes", "two abandons", "four leaves", "six misty" and "six goes" requirements, and through a series contra dictionary aesthetic concept analysis, distinguishing and rules, from different aspects, it illustrates that poems should conform to neutral specifications. "Four no" is to specify poems should achieve "degree" in "gas", "strength", "talent" and "emotion" of four aspects. And this degree is neutral, against "over" or "not much". "Two abandons" focus on meaning, but can not ignore word color, and poem meaning should direct, but cannot lack of thought. "Four leaves" require " to love from deep secluded", "by history and from the scholar", "to Manhattan from indirect" and "to fly away from the light". Six misty" criticizes six kinds of phenomena against neutralization, namely, "to the absurd is ancient, to slow is diluted, with wrong intention is only good, to strange as novelty, knowing is less stable, weak strength is easily. And the so-called "six goes" is the highest "neutral" standard for poems of Jiao Ran.

Since then, "neutralization is in theory of Confucius, Liu Xie and Jiao Ran. People focusing on literature teaching will follow Confucius saying, and saying "gentle and honest", emphasizing emotion and theory, literature and Tao, neutral literature and quality. Han Yu, Mao Kun, Li Yu and Liu Xizai are the typical representatives of this aspect. And a lot of literary people belong to the figures such as Jiao Ran and Liu Xie.

References
[1] Zhu Bokun. Learning basic course. Jiuzhou Press, 2003,2

[2] Ling Zhixuan. Mysterious Yijing. Xinjiang People's Press, 2002,10

[3] Gao Heng. "Zhouyi ancient text new notes, Chinese Book Press, 1987.

[4] Zhu Xi. Zhouyi meaning. Chinese Bookstore Press, 1987.

[5] Zhouyi research thesis collection-first series, Beijing Normal University Press, 1985.

[6] Chinese philosophy history material selection" set. Chinese Book Company, 1982.

[7] Chinese aesthetics history selection-first and second edition, Chinese Book Company, 1980.

[8] Li Zehou. Chinese ancient thought history theory. People's Press, 1986

[9] Guo Shaoyu, Wang Wensheng edited Chinese history thesis selection, Chinese Ancient Book Press, 1979.

[10] Jiang Shuge. Prose history, People's Press, 1986.

[11] Literature heritage and other materials. 